\title{
Fraktion Bündnis 90/Die Grünen im Deutschen Bundestag
}

\author{
Ulrich Schneider, MdB \\ Sprecher für Jugendpolitik und Bürgerschaftliches Engagement der \\ Bundestagsfraktion von Bündnis 90/Die Grüne |ulrich.schneider@bundestag.de \\ www.ulrich-schneider.de $\mid$ www.gruene-bundestag.de
}

Freiwilligendienste sollen Menschen die Möglichkeit eröffnen sich für einen begrenzten Zeitraum in vollem Umfang für andere einzusetzen und zu engagieren. Kleinen wie großen Organisationen soll durch

BÜNDNIS 90 DIE GRUNAN staatliche Förderung die Durchführung von Freiwilligendiensten ermöglicht werden. Gleichzeitig müssen alle Freiwilligendienste in zivilgesellschaftlicher Organisation gestärkt werden. Sie sind keine schönen Programme sondern sollen helfen lebendige Zivilgesellschaft weiter zu entwickeln, indem sie Perspektivwechsel möglich machen.

\section{Freiwilligendienste sind freiwillig}

Mittlerweile gibt es ein vielfältiges Angebot an Freiwilligendiensten. Das ist gut. Wichtig ist, dass die unterschiedlichen Formate Mindeststandards in Qualität erfüllen und tatsächlich dem Format eines Freiwilligendienstes folgen. Unser Maßstab ist deshalb: Freiwilligendienste sind freiwillig. Menschen egal welchen Alters entscheiden sich frei und aktiv für einen Dienst. Die Aufgaben müssen entsprechend flexibel und attraktiv gestaltet sein. Freiwilligendienste können für Menschen jeden Alters Zeit für Perspektivwechsel oder Neuorientierung bieten. Sie sind Lerndienste. Jeder und jede hat einen Anspruch auf qualitativ hochwertige Begleitseminare und kann in Freiwilligendiensten neue Erfahrungen sammeln. Freiwillige sollen entsprechend ihren Wünschen und Bedürfnissen beteiligt werden. Sie müssen die Möglichkeit haben, sich zu organisieren und institutionell vertreten sein. Nur wenn diese Aspekte gegeben sind, ist es tatsächlich ein echter Freiwilligendienst. Staatliche Aufgabe muss es sein, Standards zu setzen, um Missbrauch zu verhindern. Beispielsweise müssen die ehemaligen Zivildienststellen, die ohne erneute Überprüfung automatisch zu Bundesfreiwilligendienstplätzen wurden, dringend auf ihre Arbeitsmarktneutralität geprüft werden. 


\section{Freiwilligendienste - national, europäisch, international}

Freiwilligendienste geben Einblicke in die vielfältigen Facetten von Gesellschaft und Zivilgesellschaft auf nationaler, europäischer und internationaler Ebene. Deshalb sollte weniger kategorisch in einzelnen politischen Ebenen gedacht werden, sondern vielmehr national, europäisch und international ein weltumspannendes Netz gegenseitigen Austauschs entstehen. Zukünftig sollten die unterschiedlichen Dienste stärker in den europäischen und internationalen Kontext eingebettet werden. Auch in den nationalen Freiwilligendiensten muss der Austausch viel stärker in den Mittelpunkt rücken. $\mathrm{Ob}$ Freiwilliges Soziales Jahr (FSJ) oder Internationaler Jugendfreiwilligendienst (IJFD): Alle sollten gleiche Möglichkeiten des Reverse und Incoming für Freiwillige außerhalb Deutschlands bieten.

\section{Wirkung von Freiwilligendiensten}

Über viele Facetten und Wirkungsweisen von Freiwilligendiensten wissen wir noch relativ wenig. Viele der Freiwilligendienstprogramme wurden bisher unabhängig voneinander evaluiert. Insbesondere die Durchführung des Bundesfreiwilligendienst ist sehr kritisch zu sehen, wie auch eine Kurzstudie des Centrum für soziale Investitionen und Innovationen (CSI) kürzlich aufzeigte. Bis Herbst 2015 haben die Institute INBAS-Sozialforschung, INBAS (Institut für Berufliche Bildung, Arbeitsmarkt- und Sozialpolitik) und ISG (Institut für Sozialforschung und Gesellschaftspolitik) nun den Auftrag, die unterschiedlichen Dienste zu evaluieren um erstmals über einen längeren Zeitraum eine Gesamtschau der Wirkungsweisen aufzeigen zu können. Wirkung oder/und Nichtwirkung des Zivildienstes als Pflichtdienst wurde nie in Frage gestellt. Im Freiwilligendienst ist es dagegen unbedingt notwendig, Instrument und Formen, wie beispielsweise die pädagogische Begleitung, auf Wirksamkeiten zu überprüfen. Denn unser Ziel ist es, Freiwilligendienste optimal zu gestalten. Dazu gehört es, regelmäßig kritisch zu hinterfragen, wie Träger, Freiwillige und Gesellschaft tatsächlich von einem Freiwilligendienst profitieren, um diesen kontinuierlich zu verbessern. 